\title{
ANÁLISE DE IMAGENS DINÂMICA - CARACTERIZAÇÃO DA DISTRIBUIÇÃO DE TAMANHO E FORMA DE PARTÍCULAS
}

\author{
G. HAWLITSCHEK, C. ULSEN ${ }^{*}$, H. KAHN, E. A. MASINI E M.TOCCHINI \\ Depto de Eng. de Minas e de Petróleo da Escola Politécnica - Universidade de São Paulo \\ carina@Ict.poli.usp.br
}

Artigo submetido em novembro/2013 e aceito em maio/2015

DOI: $10.15628 /$ holos.2015.1781

\section{RESUMO}

A distribuição granulométrica e a caracterização da morfologia das partículas são parâmetros que exercem grande influência no comportamento de materiais particulados, que envolve setores desde a mineração até indústrias farmacêuticas, alimentícias, dentre outros Entretanto, a caracterização morfológica consiste basicamente em análises estáticas com restrições em relação ao numero de partículas caracterizadas (baixa representatividade estatística). $\mathrm{Na}$ mineração, a possibilidade de avaliar a morfologia das partículas pode ser particularmente interessante em diferentes áreas, tais como: caracterização de partículas geradas por diferentes mecanismos de cominuição (avaliação de britadores e moinhos), o efeito da morfologia das partículas na eficiência da flotação e de processos de separações minerais que dependam do comportamento cinético das partículas (hidrociclones, mesas vibratórias, espirais concentradoras, jigues). Publicações sobre a influência da morfologia das partículas em diferentes processos de beneficiamento mineral são vastas, no entanto pouca atenção vem sendo dada à sua compreensão, e as pesquisas existentes, em sua maioria, tratam a forma das partículas apenas de uma maneira qualitativa principalmente pela falta de uma técnica que determine quantitativamente tais parâmetros. Neste trabalho é apresentada uma revisão dos métodos de caracterização de tamanho e forma de partículas e três casos de aplicação da técnica de análise de imagens dinâmica.

PALAVRAS-CHAVE: morfologia de partículas, tamanho de partículas, análise de imagens dinâmica.

\section{DYNAMIC IMAGE ANALYSIS - GRAIN SIZE AND MORPHOLOGY DISTRIBUTION CHARACTERIZATION}

\begin{abstract}
The particle shape and size distribution are parameters that exerts great influence when working with particulate material, involving from mining to pharmaceutical and food industries and others. However, shape analyzes consist basically on static analyzes with strong limitations on the number of particles analyzed (low statistical significance). In the mining industry, the possibility to evaluate the particle's shape may be particularly interesting in several areas, for example: characterization of particles generated by different comminution mechanisms (evaluation of crushers and mills), the effect of particle morphology in the efficiency of flotation, processes separation and
\end{abstract}

minerals that depend on particles kinetic behavior (hydrocyclones, vibrating tables, spirals concentrators, jigs). There are several papers regarding the influence of particle morphology in various mineral beneficiation processes; however, according to the international literature little attention has been given to its understanding, and existing researches focus, mostly with particle's shape only on a qualitative approach, mainly by the lack of a technique able to quantify these parameters. This paper presents a review of characterization methods for particle size and shape and three case studies on the application of the dynamic image analysis.

KEYWORDS: particles morphology, grain size analysis, dynamic image analysis. 


\section{INTRODUÇÃO}

A distribuição granulométrica e a morfologia das partículas são parâmetros que exercem grande influência quando se trabalha com material particulado, ou seja, inúmeros setores, dos quais se destacam as indústrias de mineração, farmacêutica, alimentícia e automotiva, tintas, cosméticos, aerossóis, polímeros, dentre outras.

$\mathrm{Na}$ indústria mineral, a possibilidade de se avaliar a forma das partículas pode ser particularmente interessante em diversas áreas, como por exemplo: caracterização de partículas geradas por diferentes mecanismos de fragmentação (avaliação de britadores e moinhos), efeito da morfologia das partículas na eficiência da flotação e de operações unitárias de processamento mineral que dependam do comportamento cinético das partículas (hidrociclones, mesas vibratórias, espirais concentradoras, jigues), dentre outras.

$\mathrm{Na}$ indústria de construção civil, a distribuição granulométrica e a morfologia das partículas são fatores extremamente importantes na utilização de agregados, uma vez que influenciam diretamente no comportamento reológico do sistema e, consequentemente, no desempenho de argamassas e concretos (OLIVEIRA, STUDART et al., 2000).

Para agregados graúdos $(>4,8 \mathrm{~mm})$, a morfologia das partículas é determinada segundo procedimentos normativos (ABNT, 2008), através da medição manual do maior e menor diâmetro de cada partícula utilizando um paquímetro em amostras cujas massas mínimas variam de 9 a $32 \mathrm{~kg}$, dependendo da granulometria. Para agregados miúdos (<48 mm), devido à impossibilidade de realizar medidas com paquímetro, são utilizadas análises de forma estáticas, normatizadas pela ISO 13322-1/04 (ISO, 2004). Neste tipo de análise as partículas são dispostas sobre uma superfície plana, suportes ou lâminas e as imagens adquiridas através de microscopia óptica ou eletrônica de varredura. Esses métodos são extremamente morosos, imprecisos e possuem baixa reprodutibilidade e representatividade estatística, uma vez que dependem do operador e a aquisição de um número significativo de partículas ou imagens levaria um tempo extremamente elevado.

Atualmente, com o desenvolvimento de sistemas de análise de imagens dinâmica tem-se a possibilidade de determinação dos parâmetros geométricos através da análise de imagens obtidas por fluxo de partículas, possibilitando a caracterização de dezenas de milhares até milhões de partículas em poucos minutos. Este método de medida é descrito na norma ISO 13322-2/06 (ISO, 2006), sendo a representatividade a principal vantagem em relação aos métodos estáticos; os equipamentos disponíveis são: CAMSIZER (Retsch Technology), QICPIC (Sympatec), CPA (Haver \& Boecker) e RapidVue (Beckman Coulter).

O objetivo deste trabalho é avaliar o emprego de análise de imagens por fluxo de partículas na caracterização de amostras de areia de mesma distribuição granulométrica, e demonstrar a influência de sua forma na formulação de argamassas.

\section{MATERIAIS E MÉTODOS}

Foram analisados três tipos de materiais com características distintas através das técnicas de peneiramento e análise de imagens dinâmica. Num primeiro caso efetuou-se uma 
comparação entre as duas técnicas citadas; num segundo discute-se aspecto morfológico de produtos britados e no terceiro a influencia da morfologia dos agregados em argamassas.

O peneiramento é uma das técnicas mais antigas e utilizadas não só na indústria mineral como em diversas outras áreas (SAMPAIO, FRANÇA et al., 2007). Contudo, a análise por peneiramento de amostras com ampla distribuição é relativamente demorada. Caso haja mais de $5 \%$ em massa de material com granulometria abaixo de $37 \mu \mathrm{m}$, torna-se necessário realizar o peneiramento a úmido, o que aumenta ainda mais o tempo necessário e a eficiência é sempre prejudicada. Neste contexto, equipamentos de análise de imagens por fluxo dinâmico mostramse como uma alternativa já que alguns possuem um módulo de análise em que material fino é disperso com um jato de ar, resultando em uma distribuição granulométrica mais precisa.

Análises por sistemas de análise de imagens dinâmica foram efetuadas em equipamentos da marca Retsch, modelos Camsizer L e Camsizer XT; em ambos os equipamentos o material passa por uma área onde existe uma fonte de luz LED e duas câmeras que captam simultaneamente imagens que são processadas por um software de análise de imagens. No CAMSIZER $L$ as análises são efetuadas com o material em queda livre (material seco) em uma área restrita com limites analíticos de $30 \mu \mathrm{m}$ a $30 \mathrm{~mm}$; já no CAMSIZER-XT, que opera com material seco e também em meio líquido $(<0,6 \mathrm{~mm})$ e tem alcance de $1 \mu \mathrm{m}$ a $3 \mathrm{~mm}$.

A distribuição de tamanhos de partículas pode ser expressa tanto segundo equivalentes das séries de peneiras Tyler ou ASTM, como em quaisquer outras faixas definidas pelo usuário (até 50 classes). Os resultados podem ser expressos em termos de diferentes parâmetros de tamanho, tais como: diâmetro minimo (x_min), área equivalente (x_area) e diâmetro de Ferret máximo (Fe_max), entre outros; sendo o "x_min" o que mais se aproxima do peneiramento.

A análise das imagens possibilita também a determinação de diversos aspectos relativos a morfologia das partículas, sendo os mais utilizados esfericidade $(4 \pi$.Área / Perímetro2) e relação de aspecto (x_min/Fe_max). A relação de aspecto (b/l) é de fácil visualização, quanto menores os valores de b/l mais alongadas serão as partículas. Já a interpretação do parâmetro esfericidade, não é tão trivial; da maneira como é calculada, representa a angulosidade da partícula, ou seja a irregularidade do seu perímetro projetado. Portanto, quanto mais próximo de 1 , mais uniforme será uma superfície e quanto mais próximo de 0, mais irregular.

\subsection{Caso 1 - análise granulométrica de distribuições amplas}

Nesta etapa do trabalho foi utilizada uma amostra de argamassa, composta por areia($2,0+0,074 \mathrm{~mm})$ e cimento $(-30 \mu \mathrm{m})$. A análise no CAMSIZER foi realizada a seco com o módulo de pressão de ar, com pressão de $400 \mathrm{KPa}$; já o peneiramento foi feito a úmido nas malhas 2,38, $1,19,0,60$, e $0,074 \mathrm{~mm}$.

\subsection{Caso 2 - Características das partículas geradas por britagem em VSI}

A produção de areia artificial (gerada pela britagem de rochas) tem sido realizada por britagem de impacto vertical (VSI - Vertical Shaft Impactor) desde os anos 90. A utilização do VSI é principalmente recomendada para produção de areia de brita tipicamente abaixo de 4,8 (areia grossa) ou 2,4 $\mathrm{mm}$ (areia média), por gerar um produto com partículas mais esféricas e 
arredondadas, conferindo ao concreto melhor trabalhabilidade (CHAVES e PERES, 2006; BENGTSSON e EVERTSSON, 2008).

Para avaliação do desempenho de um britador de impacto vertical (VSI) na geração de partículas mais esféricas, uma amostra de brita, com granulometria abaixo de $19 \mathrm{~mm}$ (TQ), foi cominuída em VSI abaixo de 3,36 mm em três velocidades de rotação: $55 \mathrm{~m} / \mathrm{s}$ (VSI-55), $65 \mathrm{~m} / \mathrm{s}$ (VSI-65) e $75 \mathrm{~m} / \mathrm{s}$ (VSI-75) e, posteriormente, analisada.

\subsection{Caso 3 - Estudo preliminar da influência da morfologia das partículas em argamas-} sas

Esta etapa consistiu em avaliar o aumento do consumo de água para que argamassas com diferentes tipos de areia alcancem uma mesma consistência. Para isso, foram utilizadas areias comercializadas na cidade de São Paulo e com a mesma distribuição granulométrica. É importante garantir que todas as areias estejam com a mesma granulometria para que a diferença nos consumos de água seja decorrente das diferenças na morfologia das areias (principalmente área superficial).

As argamassas foram preparadas com a proporção, em volume, de $25 \%$ de cimento e $75 \%$ de areia e a água consumida para cada uma foi tal que proporcionasse um espalhamento de 250 $\mathrm{mm}$ no ensaio de mesa oscilatória (ABNT, 2005).

\section{RESULTADOS}

\subsection{Caso 1 - análise granulométrica de distribuições amplas}

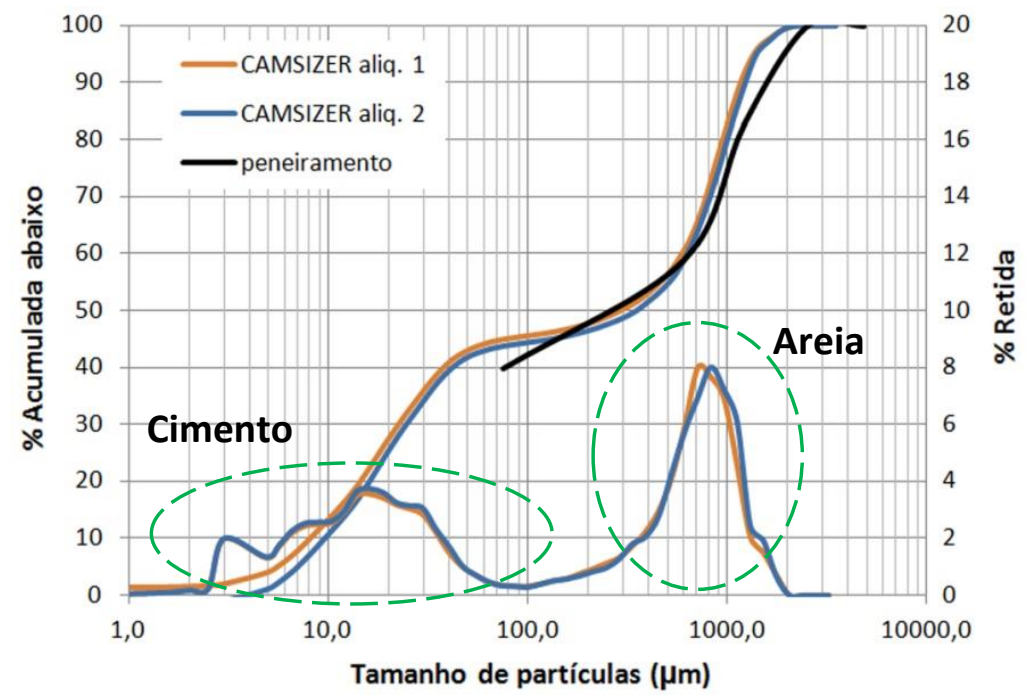

Figura 1 - Distribuição granulométrica comparativa entre análise de imagens e peneiramento de uma argamassa (areia+cimento)

A Figura 1 apresenta as distribuições granulométricas comparativas obtidas por análise de imagens dinâmica no equipamento Camsizer (parâmetro Xc_min) e por peneiramento a úmido. A semelhança dos resultados das duas técnicas é evidente, com a vantagem de o Camsizer realizar cada medida em cerca de 2 minutos, enquanto que o peneiramento, se realizado a seco, leva 
pelo menos 20 minutos, e se for a úmido em horas. Além disso, a análise de imagens permite a caracterização de partículas até a ordem de poucos micrometros, enquanto por peneiramento o limite inferior é bem maior (dezenas de micrometros).

Adicionalmente, a análise no CAMSIZER apresenta uma elevada repetitividade e com o detalhamento da curva granulométrica obtida, é possível diferenciar claramente os dois materiais presentes: areia $(>100 \mu \mathrm{m})$ e cimento $(<30 \mu \mathrm{m})$. Com isso, é possível quantificar a proporção de cada material da argamassa e, assim, realizar um controle de qualidade da formulação.

3.2 Caso 2 - Características das partículas geradas por britagem em VSI

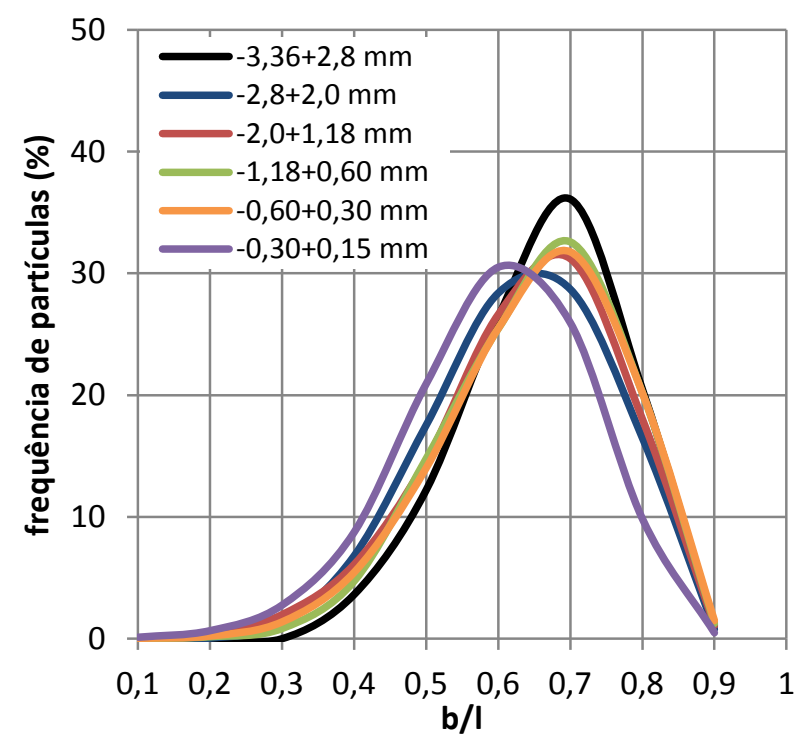

(a) TQ

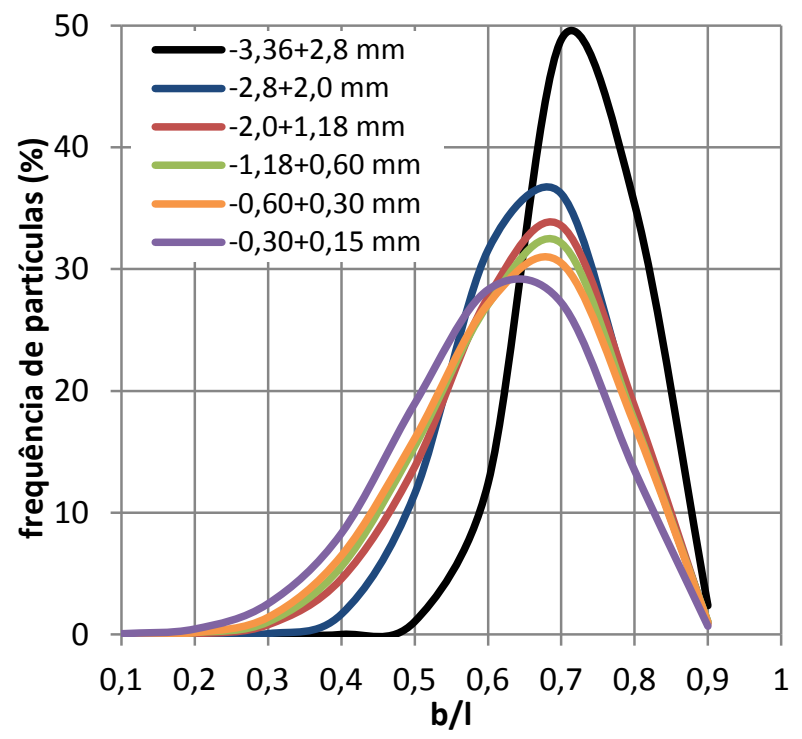

(c) VSI-65

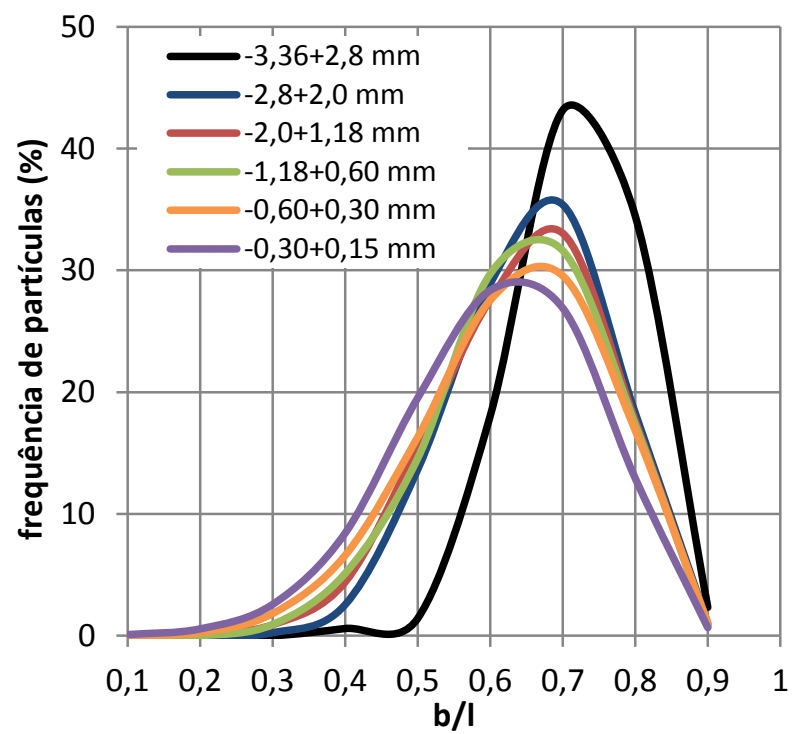

(b) VSI-55

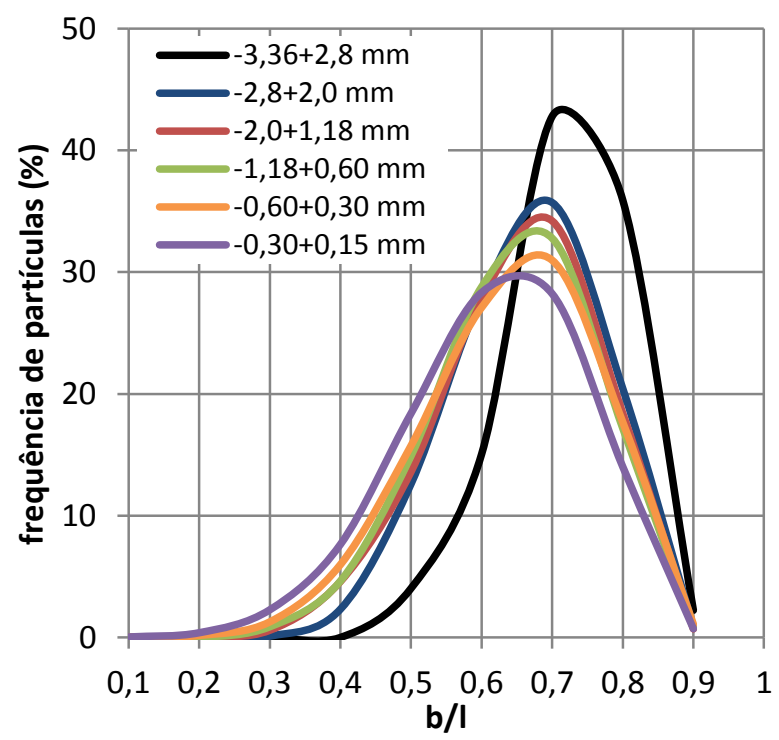

(d) VSI-75

Figura 2 - Distribuição da relação de aspecto por faixa granulométrica 
Para efeitos comparativos, são mostradas na Figura 2, as frequências de partículas com diferentes relações de aspecto por faixa granulométrica, de modo que se observa que:

- Para a amostra TQ (somente frações entre 3,36 e 0,15 mm), a relação de aspecto, dentre as diferentes faixas granulométricas, apresenta um comportamento semelhante, com uma distribuição ampla de valores (entre 0,2 e 0,9);

- Já para as amostras britadas em VSI (VSI-55, VSI-65 e VSI-75), a fração mais grossa ($3,36+2,8 \mathrm{~mm}$ ) apresenta uma distribuição bem mais estreita e com valores mais elevados (entre 0,5 e 0,9), em comparação com as demais frações. A medida que o tamanho das partículas decresce, a amplitude da distribuição da relação de aspecto aumenta, indicando que quanto menor o tamanho das partículas, maior é a presença de partículas alongadas (com baixos valores de relação de aspecto).

Essa diferença na morfologia das partículas ao longo do intervalo granulométrico pode ser explicada analisando-se o mecanismo de fragmentação no VSI. Neste tipo de britador, a quebra de partículas é feita através do impacto das mesmas contra a câmara de britagem e dentre elas próprias. Tal mecanismo de cominuicão promove a quebra das arestas das partículas mais grossas, tornando-as mais arredondadas; por outro lado, promove a geração de uma fração fina com partículas mais angulosas. Isto indica que o VSI gera uma areia arredondada, como descrito na literatura, porém, também gera partículas finas com formas mais irregulares.

3.3 Caso 3 - Estudo preliminar da influência da morfologia das partículas em argamassas

As curvas granulométricas das três areias, obtidas pelo equipamento CAMSIZER, assim como o peneiramento são ilustradas na Figura 3. Todas as areias apresentam a mesma distribuição de acordo com as 2 técnicas, portanto, a influência da granulometria na formulação da argamassa é mínima.

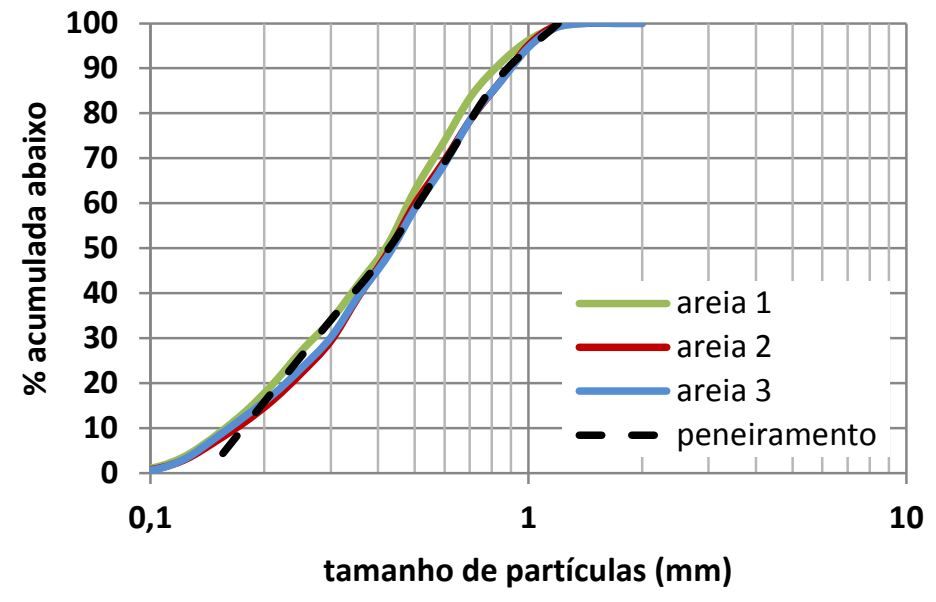

Figura 3 - Distribuição granulométrica obtida por análise de imagens das areias

A esfericidade e a relação de aspecto médio das amostras, e o teor de água das argamassas formuladas com as areias em questão, são apresentados na Tabela I. A diferença máxima de esfericidade é de 0,03 e de relação de aspecto (b/l) de 0,05. 
Tabela I - Parâmetros médios de forma e das amostras estudadas e teor de água das argamassas

\begin{tabular}{lccc}
\hline \multirow{2}{*}{ Parâmetros de forma } & \multicolumn{3}{c}{ Areia de diferentes origens } \\
\hline Esfericidade média & areia 1 & areia 2 & areia 3 \\
b/I médio & 0,807 & 0,795 & 0,826 \\
Teor de água & 0,674 & 0,710 & 0,723 \\
\hline
\end{tabular}

O teor de água, em massa, necessário para que as argamassas alcançassem a mesma consistência (espalhamento de $250 \mathrm{~mm}$ no ensaio de mesa oscilatória) aumentou para argamassas formuladas com areias mais alongadas (menor relação b/l). Ou seja, quanto menor a relação de aspecto das partículas, maior o consumo de água para que a argamassa atinja a mesma trabalhabilidade.

Tomando-se como referência a amostra mais esférica e menos alongada, areia 3, verificase que a areia 2 possui esfericidade $4 \%$ menor e relação de aspecto $2 \%$ mais baixa, o que ocasionou em um consumo de $18,5 \%$ no teor de água da argamassa (porcentagem relativa) para a mesma consistência. Para a argamassa confeccionada com amostra areia 1, com esfericidade $2 \%$ menor e relação de aspecto $7 \%$ mais baixa, foi necessário um teor de água $33 \%$ maior do que a areia 3. Tais resultados demonstram a íntima relação entre a forma das partículas e o desempenho das argamassas.

\section{DISCUSSÃO DE RESULTADOS E CONCLUSÕES}

Apesar da literatura que aborda a influência da forma das partículas nas operações de processamento mineral serem vastas, a maioria retrata a morfologia apenas de uma maneira qualitativa. A análise de imagens dinâmica apresenta-se como uma importante ferramenta, pois permite a caracterização de milhares de partículas sem interferência do operador, gerando informações com elevada representatividade e precisão estatística.

A distribuição granulométrica por peneiramento assemelha-se e à obtida por meio da análise de imagens no CAMSIZER, segundo o parâmetro "x_min". A análise de imagens dinâmica permite ainda a caracterização granulométrica e da morfologia das partículas, em poucos minutos, com elevada repetitividade e detalhamento da curva granulométrica, sendo assim possível quantificar composições de diferentes materiais e com ampla distribuição granulométrica, como no caso de argamassas.

A caracterização morfológica das partículas por intervalo granulométrico demonstrou que a britagem em VSI gera um produto com partículas grossas de elevada esfericidade, tal como descrito na literatura, mas também gera partículas finas com formas mais irregulares.

Argamassas confeccionadas com areias de distribuições granulométricas similares mostram que uma redução na relação de aspecto média de 0,05 pode resultar em um aumento de cerca de $30 \%$ no consumo de água para se atingir o mesmo espalhamento. 


\section{REFERÊNCIAS BIBLIOGRÁFICAS}

1. ABNT: NBR 13276:2005: Argamassa para assentamento e revestimento de paredes e tetos Preparo da mistura e determinação do índice de consistência. 2005.

2. _ _ _ NBR-7809: Versão Corrigida - Agregado graúdo - Determinação do índice de forma pelo método do paquímetro - Método de ensaio. 2008.

3. BENGTSSON, M.; EVERTSSON, C. M. Modelling of output and power consumption in vertical shaft impact crushers. International Journal of Mineral Processing, v. 88, n. 1-2, p. 18-23, 2008.

4. CHAVES, A. P.; PERES, A. E. C. Britagem, peneiramento e moagem. 3. São Paulo: Editora Signus, 2006. 250 p.

5. ISO. 13322-1 Particle size analysis - Image analysis methods - Part 1: Static image analysis methods. STANDARDIZATION, I. O. F.: 39 p. 2004.

6. _ـ 13322-2 Particle size analysis -- Image analysis methods -- Part 2: Dynamic image analysis methods. STANDARDIZATION, I. O. F. 2006.

7. OlIVEIRA, I. R.; STUDART, A. R.; PILEGGI, R. G.; PANDOLFELLI, V. C. Dispersão e empacotamento de partículas: princípios básicos e aplicações em processamento cerâmico. Fazendo Arte Editorial, 2000.

8. SAMPAIO, J. A.; FRANÇA, S. C. A.; BRAGA, P. F. A. Tratamento de minérios: prátricas laboratoriais. Rio de Janeiro: CETEM/MCT, 2007. 507 p. 